\title{
Near Miss Materno: vivência dos enfermeiros obstetras em maternidades de referência
}

\author{
Caroline Lima dos Reis \\ Enfermeira graduada pela Universidade Tiradentes (2017) \\ Residente em Enfermagem Obstétrica no Programa de Residência \\ Multiprofissional da Universidade Federal de Sergipe (2018-2020) \\ $\bowtie$ carolinelimareis@hotmail.com
}

Catarine Albuquerque Santana

Enfermeira graduada pela Universidade Tiradentes (2017)

\section{Lourivânia Oliveira Melo}

Enfermeira, Especialista e docente do curso de Enfermagem da Universidade Tiradentes - UNIT

\author{
Maria Inês Brandão Bocardi
}

Enfermeira, Doutora, docente do curso de Enfermagem da Universidade Tiradentes - UNIT

Max Oliveira Menezes

Enfermeiro, Especialista, docente do curso de Enfermagem da Universidade Tiradentes - UNIT

\section{Rosemar Barbosa Mendes}

Enfermeira, Mestre, docente do curso de Enfermagem da Universidade Federal de Sergipe - UFS.

Recebido em 18 de março de 2018

Aceito em 26 de julho de 2018

\section{Resumo:}

Near Miss Materno (NMM) é definido como uma mulher que quase morreu, mas sobreviveu à complicações que ocorreram durante a gravidez, parto ou até 42 dias após o fim da gestação. Nesse contexto, ressalta-se que a assistência de enfermagem para essas mulheres é complexa e, como tal, comporta inúmeras necessidades para o desenvolvimento do cuidado. A dinâmica entre os profissionais, a condição dos pacientes e a utilização de inúmeras tecnologias demandam da enfermagem conhecimentos de ordens diversas, potencializando a assistência prestada e maximizando processos efetivos de trabalho e cuidado. Esse estudo objetiva caracterizar a caracterizar a vivência do Near Miss Materno por enfermeiros obstetras atuantes nas maternidades de referência de Aracaju/Sergipe. Trata-se de um estudo exploratório, descritivo com abordagem quantitativa. Adotou-se uma amostra não probabilística intencional, a qual foi composta por 25 enfermeiros obstetras das maternidades de referência em Aracaju/SE. Os dados foram analisados de forma descritiva e analítica por meio do R-project (versão 3.4.2). A caracterização socioprofissional dos enfermeiros foi caracterizado por predomínio de idade entre 30 a 39 anos em início de carreira profissional, desenvolvimento de 40 a 60 horas semanais de trabalho e vínculo celetista. As situações de NMM foram vivenciadas por $76 \%$ desses profissionais, $56 \%$ tiveram sentimentos positivos frente a assistência desenvolvida. Os voluntários possuem vivência sobre NMM decorrente da prática profissional. No entanto, é preciso educação continuada, aumentar produção científica e exploração da temática na matriz curricular da pós-graduação.

Palavras-chave: Enfermagem, Enfermeiros Obstetras, Morbidade, Near Miss, Saúde Materna. 


\title{
Maternal Near Miss: experiences of obstetric nurses in references maternity hospitals
}

\begin{abstract}
:
Maternal Near Miss (MNM) can be defined as a woman who almost died but survived the complication that occurred during pregnancy, childbirth or up to 42 days after the end of gestation. In this context, it is emphasized that nursing care for these women is complex and, as such, entails numerous needs for the care development. The dynamics among the professionals, the patients' condition and the use of innumerable technologies require the nursing knowledge in different orders, maximizing the assistance provided, effective work and care processes. This study has the objective to characterize the experience of obstetric nurses with Maternal Near Miss in reference maternity hospitals in Aracaju / SE. This is an exploratory, descriptive study with a quantitative approach. An intentional non-probabilistic sample was used, which was composed of 25 obstetric nurses from the reference maternity hospitals in Aracaju / SE. The data were analyzed in a descriptive and analytical way using R-project (version 3.4.2). The socioprofessional characterization of nurses was defined as the predominance of the age group from 30 to 39 years old at the beginning of their professional career, the development of 40 to 60 hours a week of work and a contractual relationship. The MNM situations were experienced by $76 \%$ of these professionals, $56 \%$ had positive feelings about the developed assistance. The volunteers have experience on MNM due to professional practice. However, it is necessary to continue education, increase scientific production and explore the subject in the curricular matrix of the graduation.
\end{abstract}

Keywords: Nursing, Nurse Midwives, Morbidity, Near Miss, Maternal Health.

\section{Near Miss Materno: vivencia de los enfermeros obstetras en maternidades de referencia}

\begin{abstract}
Resumen:
Near Miss Materno (NMM) se define como una mujer que casi murió, pero sobrevivió a la complicación que ocurrió durante el embarazo, parto o hasta 42 días después del fin de la gestación. En ese contexto, se resalta que la asistencia de enfermería para esas mujeres es compleja y, como tal, comporta innumerables necesidades para el desarrollo del cuidado. La dinámica entre los profesionales, la condición de los pacientes y la utilización de innumerables tecnologías demandan de la enfermería conocimientos de órdenes diversas, potenciando la asistencia prestada y maximizando procesos efectivos de trabajo y cuidado. El estudio presenta como objetivo caracterizar la vivencia de los enfermeros obstetras con Near Miss Materno en las maternidades de referencia en Aracaju/SE. Se trata de un estudio exploratorio, descriptivo con abordaje cuantitativo. Se adoptó una muestra no probabilística intencional, la cual fue compuesta por 25 enfermeros obstetras de las maternidades de referencia en Aracaju/SE. Los datos fueron analizados de forma descriptiva y analítica a través del R-project (versión 3.4.2). La caracterización socio profesional de los enfermeros fue caracterizada por predominio de edad entre 30 a 39 años en el inicio de la carrera profesional, desarrollo de 40 a 60 horas semanales de trabajo y vínculo regido por la Consolidación de las Leyes del Trabajo (C.L.T.) brasileña. Las situaciones de NMM fueron vivenciadas por el $76 \%$ de esos profesionales, el $56 \%$ tuvo sentimientos positivos frente a la asistencia desarrollada. Los voluntarios poseen vivencia sobre NMM derivada de la práctica profesional. Sin embargo, es necesario una educación continuada, aumentar la producción científica y la exploración de la temática en la matriz curricular del postgrado.
\end{abstract}

Palabras clave: Enfermería, Enfermeras Obstetrice, Morbilidad, Near Miss Salud, Salud Materna.

\section{INTRODUÇÃO}

A morbimortalidade materna é um grave problema de saúde pública. Avaliar sua incidência constitui uma ferramenta sensível para permear o entendimento sobre mortes 
precoces e evitáveis, que em sua quase totalidade atinge as mulheres com inúmeras vulnerabilidades sociais, configurando-se como uma grave violação dos direitos humanos (BRASIL, 2012).

A mortalidade materna (MM) foi definida em 1997 pela OMS como a morte de uma mulher durante o período gestacional ou 42 dias após o término da gestação, independente da duração, localização da gravidez, relacionado ou agravado pela gestação ou por intervenções em relação a ela, com exceção as causas acidentais ou incidentais (LUZ et al., 2014; ROSENDO; RONCALLI, 2015). Pode ser classificada como direta ou indireta, sendo as diretas aquelas que ocorrem por complicações obstétricas na gravidez, no parto ou no puerpério (WORLD HEALTH ORGANIZATION, 2010). Já as indiretas são aquelas resultantes de doenças existentes antes da gravidez ou daquelas que se desenvolveram durante o período gestacional (WORLD HEALTH ORGANIZATION, 2009).

A Organização Mundial da Saúde (OMS) estima que em 2013 cerca de 289 mil mulheres no mundo morreram durante a gestação e o parto. Esse número representa um declínio de 45\% se comparado aos 523 mil óbitos em 1990, mas está distante da quinta meta dos Objetivos de Desenvolvimento do Milênio de redução de 75\% das mortes maternas até 2015 (DIAS et al., 2014).

nacional, o Brasil reduziu sua taxa de mortes maternas em $43 \%$ desde a década de 90 . No entanto, ainda é uma das menores quando comparada a outros países latino-americanos, a saber: Peru (64\%), Bolívia e Honduras (61\%), República Dominicana (57\%), Barbados (56\%), Guatemala (49\%), Equador (44\%) (BRASIL, 2014). Mulheres sobreviventes de complicações graves do ciclo gravídico puerperal, sendo as principais causas obstétricas diretas, doenças hipertensivas, hemorragias e infecções puerperais são responsáveis por $75,0 \%$ dos óbitos maternos em todo território brasileiro compartilham dos mesmos aspectos com aquelas que morrem (ROSENDO; RONCALLI, 2015). Essa semelhança levou ao desenvolvimento do conceito de Near Miss Materno (NMM) ou Morbidade Materna Grave (MMG), cuja definição foi estabelecida pela OMS, em 2007, como uma mulher que quase morreu, mas sobreviveu à complicação que ocorreu durante a gravidez, parto ou até 42 dias após o fim da gestação (WORLD HEALTH ORGANIZATION, 2010). Em 2009, a OMS propôs uma nova classificação, composta por domínios clínico, laboratorial e de manejo, com o intuito de padronizar os critérios definidores do NMM (DIAS et al., 2014; GALVÃo et al., 2014; OLIVEIRA; COSTA, 2013). 
A prevalência de NMM varia amplamente a partir dos critérios utilizados, assim foi observado a prevalência de 0,34 - 4,92 \% na América Latina e Caribe, 0,05 - 14.98 \% na África, 0,02 - 5,07 \% na Ásia, 0,04 - 0,79\% na Europa, 0,07 - 1,38 \% na América do Norte e 1,25 \% na Oceania (Austrália) (TUNÇALP et al., 2012). Pesquisa nacional observou prevalência de 21,1 casos de NMM para cada 1.000 nascidos vivos (NV) (OLIVEIRA; COSTA, 2013). Em Aracaju, no estado de Sergipe, estudo com 16.549 partos, identificou 79 casos de NMM e 17 óbitos maternos. Encontraram coeficientes de mortalidade materna, 102,7 casos/100.000 NV; prevalência de NMM, 4,7 casos/1.000 NV; razão de NMM: MM de 4,5:1 -o que indica número de casos de NMM 350\% superior ao de MM (NARDELO et al., 2017).

Estudar o NMM, especialmente em países em desenvolvimento, é fundamentalmente importante, visto as altas taxas apresentadas, como por exemplo, 10,2 casos de NMM por mil NV e uma razão de mortalidade do NMM de 30,8 casos para cada MM, sendo estimados mais de 23 mil casos na amostra expandida para o país, segundo o estudo Nascer no Brasil (DIAS et al., 2014). Além disso, podem retratar a peculiaridade da assistência prestada à saúde da mulher (WORLD HEALTH ORGANIZATION, 2009).

Nesse contexto, salienta-se que o trabalho da enfermagem com pacientes críticos é complexo e, como tal, comporta inúmeras necessidades para desenvolvimento do cuidado. A dinâmica entre os profissionais, a condição dos pacientes e a utilização de inúmeras tecnologias demandam da enfermagem conhecimentos de ordens diversas, potencializando a assistência prestada e maximizando processos efetivos de trabalho e cuidado (MASSAROLI et al., 2015). O enfermeiro obstetra tem papel fundamental no desenvolvimento dessa assistência, pois é um dos primeiros a identificar qualquer alteração que ocorra com a mulher durante o ciclo gravídico puerperal (DIAS et al., 2014; FEITOSA; LEITE; SILVA, 2012; COFEN, 2016).

Deste modo, esse estudo apresenta significativa importância para o cenário obstétrico local, contribuindo para a melhoria da assistência desenvolvida. Agrega-se valor, por também auxiliar os gestores no desenvolvimento de protocolos e cursos de aperfeiçoamento, provimento de dados que podem contribuir com a formação acadêmica de enfermagem e especialistas em enfermagem obstétrica, culminado na redução da morbimortalidade materna. 
Além disso, é um tema de grande magnitude na pauta sobre a assistência à gravidez, parto e puerpério, pois mesmo com sua importância ainda há escassa produção científica acerca da temática no Brasil, especialmente voltado ao especialista.

Desta forma o trabalho tem como objetivo geral caracterizar a vivência do Near Miss Materno por enfermeiros obstetras atuantes nas maternidades de referência de Aracaju/Sergipe.

\section{METODOLOGIA}

Trata-se de um estudo exploratório, descritivo com abordagem quantitativa. 0 trabalho foi desenvolvido nas duas maternidades de referência da cidade de Aracaju, em Sergipe no período de outubro a novembro de 2017.

A Maternidade Nossa Senhora de Lourdes - MNSLé uma unidade de alta complexidade vinculada ao Sistema Único de Saúde (SUS), referência estadual para o atendimento de gestantes de alto risco, ofertando serviços de urgência e emergência obstétrica com implementação da classificação de risco (SERGIPE, 2017). A Maternidade Santa Isabel - MSI apresenta gestão de ordem filantrópica e convênio com o Sistema Único de Saúde, destinando assistência as gestantes de risco habitual. Ambas instituições apresentam assistência de enfermagem 24 horas, com notabilidade de enfermeiros especialistas em Enfermagem Obstétrica. Estima-se que as duas instituições são provedoras de assistência obstétrica para uma população de aproximadamente dois milhões de pessoas que incluem toda a população do estado e algumas regiões dos estados vizinhos (IBGE, 2010).

\section{Critérios de Inclusão e Exclusão; População e Amostra;}

Foram incluídos no estudo os enfermeiros obstetras atuantes nas duas maternidades de referência em Aracaju, mediante anuência com Termo de Consentimento Livre e Esclarecido (TCLE), e excluídos todos os seis profissionais em que: na ocasião da coleta de dados estavam em gozo de férias ou afastamento laboral, em processo de especialização em enfermagem obstétrica e envolvidos com a produção do estudo.

A população foi composta por 31 enfermeiros obstetras de ambas maternidades. Assim, a amostra culminou em 25 voluntários (Figura 1). 
Figura 1: Distribuição dos enfermeiros obstetras constituintes da população/ amostra.

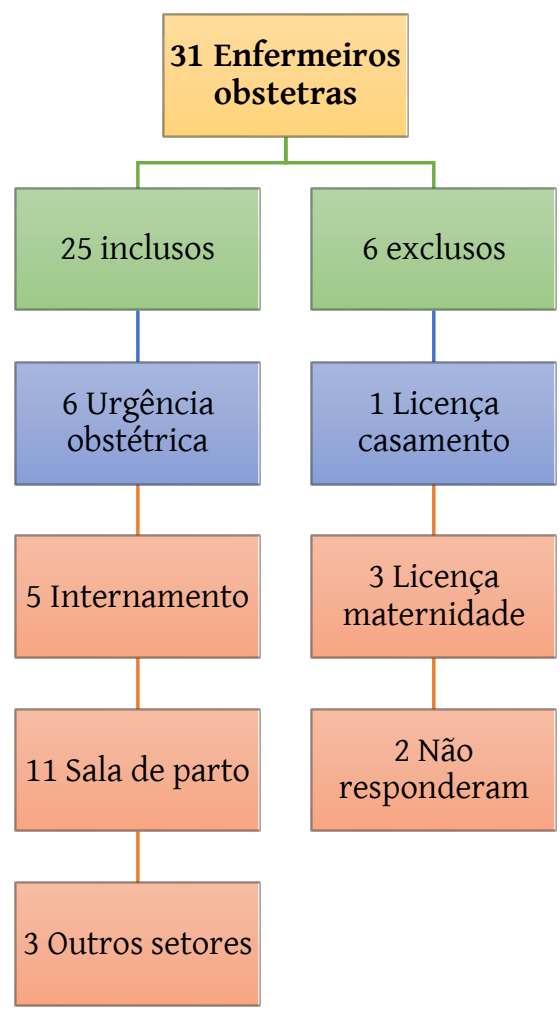

Fonte: Dados da pesquisa, 2017.

\section{Instrumentos de Coleta de Dados}

Os dados foram coletados por meio de um questionário estruturado com questões fechadas, elaborado pelos pesquisadores, salienta-se que o mesmo foi submetido a avaliação prévia de enfermeiros (as) especialistas e com experiência profissional em Enfermagem obstétrica.

Este instrumento foi composto por três partes, totalizando 19 questões. A primeira é destinada a identificação socioprofissional do público alvo. A segunda parte do instrumento diz respeito aos critérios para definição e abordagem ao NMM (critérios validados e publicados pela OMS). E a última parte do instrumento diz respeito a caracterização da assistência de enfermagem e vivência profissional com NMM.

\section{Análise dos Dados e Estatística}

Os dados foram analisados de forma descritiva e analítica. As variáveis numéricas foram observadas quanto à distribuição de normalidade por meio do teste de Shapiro-Wilk. 
Nos casos em que os pressupostos foram atendidos, as mesmas foram apresentadas por meio de média e desvio-padrão. As variáveis categóricas serão apresentadas por meio de frequência absoluta e relativa. O programa estatístico utilizado foi o R-project (versão 3.4.2).

\section{Aspectos Éticos}

A pesquisa teve início mediante anuência do Comitê de Ética em Pesquisa com o parecer consubstanciado $n^{\circ} 2.317 .557$. Na posse da autorização, os pesquisadores seguiram o fluxo: Identificação dos enfermeiros obstetras atuantes nas instituições pesquisadas; busca ativa dos enfermeiros em horário laboral no decorrer das semanas; apresentação dos pesquisadores, explicação e leitura dos objetivos da pesquisa; apresentação do questionário; apresentação e leitura do Termo de Consentimento Livre e Esclarecido (TCLE) para o profissional e solicitação da assinatura em duas vias; aplicação do questionário de acordo com disponibilidade do enfermeiro. Os voluntários foram informados que receberão o resultado final da pesquisa. Está em consonância com a Resolução 466/2012 do Conselho Nacional de Saúde, que fala sobre as diretrizes e normas regulamentadoras de pesquisas envolvendo seres humanos. Ressaltamos ainda, o compromisso por parte de todos os pesquisadores envolvidos na manutenção do anonimato e sigilo das informações, destinando-as exclusivamente para fins científicos. O material coletado ficará sob a guarda do pesquisador responsável por um período de 5 anos.

\section{RESULTADOS}

A pesquisa foi realizada com 25 enfermeiros obstetras voluntários. Quanto à caracterização socioprofissional foi possível observar que estes 52\% possuíam idade entre 31 e 39 anos (média 39 anos e 6 meses, \pm 6,27 anos). No qual, 56\% viviam com o companheiro, $56 \%$ estavam em início de carreira. Predominou o vínculo celetista e carga horaria semanal entre 40 a 60 horas (tabela 1).

Tabela 1 - Caracterização socioprofissional dos enfermeiros obstetras atuantes nas duas maternidades de referência em Aracaju/SE 2017.

\begin{tabular}{c|ccc}
\hline VARIÁVEL & OPÇÃO & FREQUÊNCIA & PERCENTUAL (\%) \\
\hline \multirow{2}{*}{ Situação conjugal } & Com companheiro & 14 & 56 \\
\cline { 2 - 4 } & Sem companheiro & 11 & 44 \\
\hline
\end{tabular}




\begin{tabular}{|c|c|c|c|}
\hline \multirow{2}{*}{ Cidade } & Aracaju & 22 & 88 \\
\hline & Interior & 3 & 12 \\
\hline \multirow{3}{*}{ Tempo de atuação } & 0 a 5 anos & 14 & 56 \\
\hline & 6 a 10 anos & 8 & 32 \\
\hline & 11 a 15 anos & 3 & 12 \\
\hline \multirow{3}{*}{ Vínculo trabalhista } & CLT & 19 & 76 \\
\hline & Estatutário & 4 & 16 \\
\hline & Outros & 2 & 8 \\
\hline \multirow{2}{*}{$\begin{array}{c}\text { Número de instituições } \\
\text { que trabalha }\end{array}$} & Uma & 7 & 28 \\
\hline & Duas & 18 & 72 \\
\hline \multirow{4}{*}{ Horas semanais } & Não respondeu & 3 & 12 \\
\hline & Até 36 horas & 8 & 32 \\
\hline & 40 a 60 horas & 9 & 36 \\
\hline & Mais que 60 horas & 5 & 20 \\
\hline
\end{tabular}

Fonte: Dados da pesquisa, 2017.

No que diz respeito a vivência do NMM por parte dos enfermeiros obstetras atuantes nas duas maternidades de referência em Aracaju foi possível observar que 76\% já vivenciaram alguma situação na prática laboral. Com relação ao fluxo assistencial, observou-se homogeneidade das respostas, sendo 32\% com permanência na unidade inicial sem assistência em Unidade de Terapia Intensiva (UTI) e posterior alta, 20\% internação na UTI com subsequente alta e $24 \%$ ambas as opções de desfecho (Tabela 2).

Relacionado a vivência dos colaboradores à temática de estudo, foi possível perceber prevalência de sentimentos positivos em relação a sua prática assistencial, onde $24 \%$ assinalaram sentimento de liderança, $24 \%$ satisfação e $8 \%$ confiança. Porém, é preciso salientar que $20 \%$ dos voluntários relataram sentimentos negativos, sendo este $1 / 5$ da parcela dos pesquisados (Tabela 2).

Sobre a adequação institucional para atuação nos casos de NMM (tabela 2), 28\% dos colaboradores relataram a escassez de educação continuada sobre o assunto, $4 \%$ insumos e 56\% assinalaram mais de uma opção do questionário (aparelhos com tecnologia de grande porte, equipe multiprofissional, aporte emocional, educação continuada e insumos).

A necessidade de reformulação na matriz curricular da especialização foi apontada por $96 \%$ dos enfermeiros obstetras, destaca-se que 36\% destes assinalaram mais de uma especificidade (simulação realística, conteúdo sobre o tema, preparação psicoemocional e divulgação da temática) (tabela 2). 
Tabela 2 - Caracterização da vivência dos enfermeiros obstetras com Near Miss Materno nas duas maternidades referência de Aracaju/SE, 2017.

\begin{tabular}{|c|c|c|c|}
\hline VARIÁVEL & OPÇÃO & FREQUÊNCIA & PERCENTUAL (\%) \\
\hline \multirow{2}{*}{$\begin{array}{c}\text { Já vivenciou em sua } \\
\text { prática profissional o } \\
\text { NMM }\end{array}$} & Sim & 18 & 72 \\
\hline & Não & 7 & 28 \\
\hline \multirow{4}{*}{$\begin{array}{l}\text { Desfecho materno na } \\
\text { situação de NMM } \\
\text { vivenciada }\end{array}$} & Não respondeu & 6 & 24 \\
\hline & $\begin{array}{l}\text { Permanência na unidade } \\
\text { inicial sem assistência em } \\
\text { UTI e posterior alta. }\end{array}$ & 8 & 32 \\
\hline & $\begin{array}{l}\text { Internação na UTI com } \\
\text { subsequência alta }\end{array}$ & 5 & 20 \\
\hline & Ambas opções & 6 & 24 \\
\hline \multirow{5}{*}{$\begin{array}{l}\text { Qual seu sentimento } \\
\text { frente a uma situação } \\
\text { de NMM }\end{array}$} & Não respondeu & 6 & 24 \\
\hline & Líder & 6 & 24 \\
\hline & Satisfeito & 6 & 24 \\
\hline & Confiante & 2 & 8 \\
\hline & $\begin{array}{l}\text { Sentimento negativo } \\
\text { (despreparado, choroso, } \\
\text { impotente, confuso, } \\
\text { perdido) }\end{array}$ & 5 & 20 \\
\hline \multirow{4}{*}{$\begin{array}{l}\text { O que falta no serviço } \\
\text { para atuação eficaz } \\
\text { em situações de NMM }\end{array}$} & Educação continuada & 7 & 28 \\
\hline & Insumos & 1 & 4 \\
\hline & $\begin{array}{c}\text { Mais de um item (aparelhos } \\
\text { com tecnologia de grande } \\
\text { porte, equipe } \\
\text { multiprofissional, aporte } \\
\text { emocional, educação } \\
\text { continuada, insumos) }\end{array}$ & 14 & 56 \\
\hline & $\begin{array}{l}\text { Nenhuma das } \\
\text { Alternativa (desconsidera a } \\
\text { necessidade de mudanças) }\end{array}$ & 3 & 12 \\
\hline \multirow{5}{*}{$\begin{array}{l}\text { O que falta na matriz } \\
\text { curricular da } \\
\text { especialização } \\
\text { para subsidiar a } \\
\text { preparação do } \\
\text { enfermeiro no } \\
\text { contexto do NMM }\end{array}$} & Simulação Realística & 3 & 12 \\
\hline & Conteúdo sobre o tema & 5 & 20 \\
\hline & Divulgação da temática & 3 & 12 \\
\hline & $\begin{array}{l}\text { Todos os itens (simulação } \\
\text { realística, conteúdo sobre o } \\
\text { tema, preparação } \\
\text { psicológica e emocional, } \\
\text { divulgação da temática) }\end{array}$ & 4 & 16 \\
\hline & $\begin{array}{l}\text { Mais de um (simulação } \\
\text { realística, conteúdo sobre o } \\
\text { tema, preparação } \\
\text { psicológica e emocional, } \\
\text { divulgação da temática) }\end{array}$ & 9 & 36 \\
\hline
\end{tabular}


Nenhuma das alternativas

(desconsidera necessidade

de mudanças)

Fonte: Dados da pesquisa, 2017.

Considerando a especificidade do assunto, 64\% sabiam o conceito da OMS e $48 \%$ a avaliação correta de abordagem do NMM. Este fato, tornou-se possível a partir da vivência dos profissionais na enfermagem obstétrica, pois $72 \%$ afirmaram nunca ter lido ou estudado sobre a temática.

As mulheres em situações de NMM demandam atenção por serem pacientes de cuidados intensivos, deste modo a assistência do enfermeiro, no âmbito técnico ou gerencial, foi mais apontada para: 76\% administração de medicações, 76\% investigação laboratorial, 76\% monitorização, $72 \%$ cuidados com vias aéreas, $72 \%$ suporte e cuidado aos familiares e pacientes, 72\% evolução da assistência de enfermagem em todos os turnos e $68 \%$ tarefas administrativas e gerenciais (tabela 3).

Tabela 3- Assistência de enfermagem desenvolvida em situação de Near Miss Materno pelos enfermeiros obstetras nas duas maternidades referência de Aracaju/SE, 2017.

\begin{tabular}{|c|c|c|c|}
\hline VARIÁVEL & OPÇÃO & FREQUÊNCIA & PERCENTUAL (\%) \\
\hline \multirow[t]{3}{*}{$\begin{array}{l}\text { Administração de } \\
\text { hemoderivados }\end{array}$} & $\begin{array}{l}\text { Não respondeu ao } \\
\text { item (por não } \\
\text { vivenciar o NMM) }\end{array}$ & 6 & 24 \\
\hline & Sim & 16 & 64 \\
\hline & Não & 3 & 12 \\
\hline \multirow[t]{3}{*}{ Administração de medicações } & $\begin{array}{l}\text { Não respondeu ao } \\
\text { item (por não } \\
\text { vivenciar o NMM) }\end{array}$ & 6 & 24 \\
\hline & Sim & 19 & 76 \\
\hline & Não & 0 & 0 \\
\hline \multirow{3}{*}{$\begin{array}{l}\text { Assistência em parada } \\
\text { cardiorrespiratória }\end{array}$} & $\begin{array}{l}\text { Não respondeu ao } \\
\text { item(por não } \\
\text { vivenciar o NMM) }\end{array}$ & 6 & 25 \\
\hline & Sim & 9 & 37,5 \\
\hline & Não & 9 & 37,5 \\
\hline \multirow[t]{3}{*}{ Cuidado com drenos } & $\begin{array}{l}\text { Não respondeu ao } \\
\text { item (por não } \\
\text { vivenciar o NMM) }\end{array}$ & 6 & 24 \\
\hline & Sim & 5 & 20 \\
\hline & Não & 14 & 56 \\
\hline
\end{tabular}




\begin{tabular}{|c|c|c|c|}
\hline \multirow[t]{3}{*}{ Cuidados com vias áreas } & $\begin{array}{l}\text { Não respondeu ao } \\
\text { item (por não } \\
\text { vivenciar o NMM) }\end{array}$ & 6 & 24 \\
\hline & Sim & 18 & 72 \\
\hline & Não & 1 & 4 \\
\hline \multirow{3}{*}{$\begin{array}{c}\text { Evolução da assistência de } \\
\text { enfermagem em todos os } \\
\text { turnos }\end{array}$} & $\begin{array}{l}\text { Não respondeu ao } \\
\text { item (por não } \\
\text { vivenciar o NMM) }\end{array}$ & 6 & 24 \\
\hline & Sim & 18 & 72 \\
\hline & Não & 1 & 4 \\
\hline \multirow{3}{*}{$\begin{array}{c}\text { Implementação de balanço } \\
\text { hídrico }\end{array}$} & $\begin{array}{l}\text { Não respondeu ao } \\
\text { item (por não } \\
\text { vivenciar o NMM) }\end{array}$ & 6 & 24 \\
\hline & Sim & 10 & 40 \\
\hline & Não & 9 & 36 \\
\hline \multirow[t]{3}{*}{ Investigação laboratorial } & $\begin{array}{l}\text { Não respondeu ao } \\
\text { item (por não } \\
\text { vivenciar o NMM) }\end{array}$ & 6 & 24 \\
\hline & Sim & 19 & 76 \\
\hline & Não & 0 & 0 \\
\hline \multirow{3}{*}{$\begin{array}{c}\text { Mobilização e posicionamento } \\
\text { no leito }\end{array}$} & $\begin{array}{l}\text { Não respondeu ao } \\
\text { item (por não } \\
\text { vivenciar o NMM) }\end{array}$ & 6 & 24 \\
\hline & Sim & 16 & 64 \\
\hline & Não & 3 & 12 \\
\hline \multirow[t]{3}{*}{ Monitorização } & $\begin{array}{l}\text { Não respondeu ao } \\
\text { item (por não } \\
\text { vivenciar o NMM) }\end{array}$ & 6 & 24 \\
\hline & Sim & 19 & 76 \\
\hline & Não & 0 & 0 \\
\hline \multirow{3}{*}{$\begin{array}{c}\text { Preparação/ manutenção de } \\
\text { drogas vasoativas }\end{array}$} & $\begin{array}{l}\text { Não respondeu ao } \\
\text { item (por não } \\
\text { vivenciar o NMM) }\end{array}$ & 6 & 24 \\
\hline & Sim & 17 & 68 \\
\hline & Não & 2 & 8 \\
\hline \multirow{3}{*}{$\begin{array}{l}\text { Presença à beira do leito e } \\
\text { observação ou atividade } \\
\text { contínua } \\
\text { por uma hora ou mais, por } \\
\text { razões de segurança, } \\
\text { gravidade ou } \\
\text { terapia. }\end{array}$} & $\begin{array}{l}\text { Não respondeu ao } \\
\text { item (por não } \\
\text { vivenciar o NMM) }\end{array}$ & 6 & 24 \\
\hline & $\operatorname{Sim}$ & 15 & 60 \\
\hline & Não & 4 & 16 \\
\hline \multirow{3}{*}{$\begin{array}{c}\text { Realização de procedimentos } \\
\text { de higiene }\end{array}$} & $\begin{array}{l}\text { Não respondeu ao } \\
\text { item (por não } \\
\text { vivenciar o NMM) }\end{array}$ & 6 & 24 \\
\hline & Sim & 15 & 60 \\
\hline & Não & 4 & 16 \\
\hline
\end{tabular}




\begin{tabular}{|c|c|c|c|}
\hline \multirow[t]{3}{*}{ Sinais vitais de $15 / 15$ minutos } & $\begin{array}{l}\text { Não respondeu ao } \\
\text { item (por não } \\
\text { vivenciar o NMM) }\end{array}$ & 7 & 28 \\
\hline & Sim & 12 & 48 \\
\hline & Não & 6 & 24 \\
\hline \multirow[t]{3}{*}{ Sinais vitais de $4 / 4$ horas } & $\begin{array}{l}\text { Não respondeu ao } \\
\text { item (por não } \\
\text { vivenciar o NMM) }\end{array}$ & 8 & 32 \\
\hline & Sim & 10 & 40 \\
\hline & Não & 7 & 28 \\
\hline \multirow[t]{3}{*}{ Sinais vitais de $6 / 6$ horas } & $\begin{array}{l}\text { Não respondeu ao } \\
\text { item (por não } \\
\text { vivenciar o NMM) }\end{array}$ & 8 & 32 \\
\hline & Sim & 7 & 28 \\
\hline & Não & 10 & 40 \\
\hline \multirow{3}{*}{$\begin{array}{l}\text { Suporte e cuidado aos } \\
\text { familiares e pacientes }\end{array}$} & $\begin{array}{l}\text { Não respondeu ao } \\
\text { item (por não } \\
\text { vivenciar o NMM) }\end{array}$ & 6 & 24 \\
\hline & Sim & 18 & 72 \\
\hline & Não & 1 & 4 \\
\hline \multirow[t]{3}{*}{ Suporte para alimentação } & $\begin{array}{l}\text { Não respondeu ao } \\
\text { item (por não } \\
\text { vivenciar o NMM) }\end{array}$ & 6 & 24 \\
\hline & Sim & 9 & 36 \\
\hline & Não & 10 & 40 \\
\hline \multirow[t]{3}{*}{ Suporte ventilatório } & $\begin{array}{l}\text { Não respondeu ao } \\
\text { item (por não } \\
\text { vivenciar o NMM) }\end{array}$ & 6 & 24 \\
\hline & Sim & 14 & 56 \\
\hline & Não & 5 & 20 \\
\hline \multirow{3}{*}{$\begin{array}{c}\text { Tarefas administrativas e } \\
\text { gerenciais }\end{array}$} & $\begin{array}{l}\text { Não respondeu ao } \\
\text { item (por não } \\
\text { vivenciar o NMM) }\end{array}$ & 7 & 28 \\
\hline & Sim & 17 & 68 \\
\hline & Não & 1 & 4 \\
\hline
\end{tabular}

Fonte: Dados da pesquisa, 2017.

\section{DISCUSSÃO}

O perfil socioprofissional dos enfermeiros desse estudo apresentou consonância com outras pesquisas brasileiras (GARDENAL et al., 2002; BARBOSA; CARVALHO; OLIVEIRA, 2008), 
os quais caracterizaram-se por profissionais com idade entre 24 e 37 anos, casados, atuação em 2 ou mais instituições de saúde, com 2 ou mais anos de formação.

Nesse contexto, é possível inferir que a maturidade profissional é essencial para a compreensão do processo de trabalho e da melhoria da assistência prestada as usuárias (ESSER; MAMEDE; MAMEDE, 2012). No entanto, a qualidade assistencial pode ser comprometida por sobrecarga de trabalho, além de favorecer adoecimento mental e/ou físico, absenteísmos, acidentes de trabalho, erros de medicação, exaustão, ausência de lazer (VILLELA et al., 2011).

No que diz respeito a vivência dos enfermeiros obstetras com situações de NMM, não foi encontrado na literatura científica por meio de pesquisa nas bases de dados: Biblioteca Virtual de Saúde (BVS), Scientific Eletronic Library Online (SCIELO), Banco de Dados em Enfermagem (BDENF) e Literatura Latino-Americana e do Caribe em Ciências da Saúde (LILACS) dados plausíveis para comparação/discussão, as quais apresentam amplas publicações de estudos realizados por enfermeiros. Esse fato reafirma a necessidade de pesquisas envolvendo a temática e atuação do enfermeiro, visto que nos dias atuais existe um fortalecimento da assistência prestada por esse profissional mediante prerrogativas da Rede Cegonha.

Quanto ao desfecho materno relatado pelos voluntários deste estudo foi possível constatar não conformidade com uma pesquisa na cidade de Maringá/Paraná (DALRI et al., 2014), onde foram identificados 16 casos de NMM, destes 76\% dos internamentos cursaram com permanência na UTI com posterior alta e uma média de 4 dias de internação.

No entanto esses resultados não podem ser generalizados, uma vez que, os internamentos em UTI são influenciados por diversos fatores, destacando a condição de saúde do paciente e disponibilidade de vaga na UTI. Como exemplo, no Texas, é observada a mais alta taxa de internamento em UTI dos Estados Unidos (EUA), o qual é justificado por critérios com limiares marcadamente baixos para o internamento nesse setor, como evidenciado pelos raros casos de MM entre as internadas em UTI (0,3\%) (OLIVEIRA; COSTA, 2015). Em relação ao Brasil, a região Sul apresenta a menor taxa de internamento por complicações obstétricas, situação contraditória a região Nordeste e o Centro-Oeste com maiores taxas (RUDEY; CORTEZ; YAMAGUCHI, 2017). 
No âmbito "satisfação do enfermeiro para prática laboral" acredita-se ser de relevante contribuição para saúde mental e física deste colaborador e, assim, melhora a qualidade de vida no exercício profissional e, por consequência, propicia uma melhor assistência de enfermagem aos clientes. Pesquisas desenvolvidas com trabalhadores de enfermagem de duas UTI do serviço público mostrou que o trabalho quando é desenvolvido em equipe proporciona sentimentos de satisfação (OUD, 2017; SILVEIRA et al., 2012).

Em uma maternidade de Sorocaba/São Paulo (GARDENAL et al., 2012), 73\% das enfermeiras entrevistadas, sentiram-se satisfeita no trabalho que realizavam. Em um estudo, com enfermeiros que atuam no cuidado ao paciente crítico (MORAIS et al., 2016), observaram que os mesmos vivenciam felicidade, satisfação e gratificação quando o resultado das intervenções realizadas proporciona recuperação do paciente.

Assim é possível salientar que os sentimentos positivos do enfermeiro, oriundos da prática profissional, podem estar atrelados ao conhecimento científico e vivência de experiências práticas, visto que, quando encontra-se diante de uma nova situação de dificuldade terá subsídios para direcionar a equipe de enfermagem a uma assistência adequada e de qualidade.

Agrega-se também para uma prática laboral de qualidade a necessidade de suporte e estrutura física, humana e intelectual, que tornem melhor, as condições de trabalho e assim obtenha uma redução da morbimortalidade materna. Desde modo, são apontados em pesquisas nacionais (GARDENAL et al., 2002; OLIVEIRA; SPIRI, 2011) alguns desafios para a prática em obstetrícia como: falta de materiais hospitalares adequados para a assistência, a estrutura física, o relacionamento interpessoal e a falta da educação permanente. Esses fatores impactam negativamente na identificação e abordagem ao NMM.

Tais apontamentos reafirmam a discrepância existente entre as políticas públicas e a realidade das maternidades brasileiras, pois mesmo com a política Rede Cegonha (BRASIL, 2011), atual incentivadora para inserção e protagonismo dos enfermeiros obstetras na assistência ao ciclo gravídico puerperal, ainda está aquém do desejado o desenvolvimento profissional e das instituições (NASCIMENTO et al., 2014), fato que impacta na redução da morbimortalidade materna. 
Ficou evidente nesse estudo a necessidade de reformulação da matriz curricular em obstetrícia para novos seguimentos, como por exemplo, o NMM. Pesquisa realizada no estado de São Paulo, obteve relatos de insegurança por parte dos enfermeiros em suas atividades obstétricas; consideraram o conteúdo da pós-graduação deficiente, não atendendo expectativas e necessidades (BARBOSA; CARVALHO; OLIVEIRA, 2008). Essas fragilidades são agravadas pelo descompasso entre o tempo de abordagem dos saberes, ou seja, primeiro aprendem a teoria, para depois irem para o campo de estágio (LIMA et al., 2017)

É preciso na matriz curricular das especializações, inserir o NMM em paralelo a realização de simulação realística para o desenvolvimento de competências gerenciais e assistências, bem como ensino aprendizagem teórico/prático no combate da morbimortalidade materna, o que propicia uma melhor preparação psicoemocional destes profissionais.

Mediante os resultados observou-se o predomínio de procedimentos assistências realizados pelos enfermeiros obstetras do estudo, tal fato corrobora para necessidade de práticas privativas do enfermeiro, sendo este o profissional responsável pelos cuidados de enfermagem de maior complexidade técnica e que exijam conhecimentos de base cientifica e capacidade de tomar decisões imediatas (COFEN, 2015), adotando os procedimentos que entender imprescindíveis para garantir segurança da mulher (COFEN, 2016). Sendo assim, esses especialistas nos serviços de obstetrícia, tem como responsabilidade o acompanhamento da mulher sob seus cuidados, desde o momento da internação até a alta hospitalar (COFEN, 2016), afim de reduzir a morbimortalidade materna.

\section{CONSIDERAÇÕES FINAIS}

Ficou perceptível que a experiência culminou de forma positiva na percepção do enfermeiro sobre o NMM, pois a maioria dos enfermeiros obstetras que atuam nas maternidades de referência de Aracaju/SE já vivenciaram uma situação de NMM durante sua prática profissional. Destaca-se a necessidade de inserção da temática dentro dos cursos de pós-graduação, pois o enfermeiro obstetra encontra-se em contato contínuo com a mulher durante o ciclo gravídico puerperal, sendo este um profissional fundamental na redução das taxas de morbimortalidade materna. 
Assim, o conhecimento derivado desse estudo, poderá contribuir para o aperfeiçoamento de programas acadêmicos e institucionais voltado à temática abordada nesse estudo, bem como divulgação da mesma para comunidade científica principalmente direcionada ao especialista. Bem como, servir para delinear pesquisas futuras no âmbito da saúde da mulher, principalmente voltada a morbimortalidade materna.

\section{REFERÊNCIAS}

BARBOSA, P.G; CARVALHO, G.M; OLIVEIRA, L.R. Obstetrical nursing: discovering advantages and difficulties faced by specialists in this area. Revis. O Mundo da Saúde. v.32; n. 4, p. 458-465. São Paulo, 2008. Disponível em < https://www.saocamilo-sp.br/pdf/mundo_saude/65/07_Enfermagem_baixa.pdf> Acesso em: 25/06/2017.

BRASIL. Ministério da Saúde. Portaria no 1.459, de 24 de junho de 2011. Institui no âmbito do Sistema Único de Saúde - SUS - a Rede Cegonha. Diário Oficial [da] União, Brasília, DF, 27 jun. 2011. Seção 1, p. 109. Disponível em < http://bvsms.saude.gov.br/bvs/saudelegis/gm/2011/prt1459_24_06_2011.html> Acesso em: 16/07/2017.

BRASIL. ONU. (Org.). Mortalidade materna cai 55\% no Brasil, aponta relatório sobre Objetivos do Milênio. 2014.

BRASIL. Uma análise da situação de saúde e a vigilância da Saúde da Mulher. Ministério da Saúde, Brasília, DF 2012.

CONSELHO FEDERAL DE ENFERMAGEM. Dispõe sobre a atuação de Enfermeiros na assistência às gestantes, parturientes e puérperas. Resolução Cofen nº 477/2015. Brasília - Distrito Federal, 2015. Disponível em < http://www.cofen.gov.br/resolucao-cofen-no-04772015_30967.html> Acesso em 23/08/2017.

CONSELHO FEDERAL DE ENFERMAGEM. Normatiza a atuação e a responsabilidade do Enfermeiro, Enfermeiro Obstetra e Obstetriz na assistência às gestantes, parturientes, puérperas e recém-nascidos os Serviços de Obstetrícia, Centros de Parto Normal e/ou Casas de Parto e outros locais onde ocorra essa assistência; estabelece critérios para registro de títulos de Enfermeiro Obstetra e Obstetriz no âmbito do Sistema Cofen/Conselhos Regionais de Enfermagem, e dá outras providências. Resolução COFEN no 0516/2016. Brasília- Distrito Federal. p. 1-7, 2016. Disponível em < http://www.cofen.gov.br/resolucao-cofen-no05162016_41989.html> Acesso em: 23/08/2017.

DALRI, R.C.M.B; SILVA, L.A; MENDES, A.M.O.C; ROBAZZI, M.L.C.C. Carga horária de trabalho dos enfermeiros e sua relação com as reações fisiológicas do estresse. Rev. Latino-Am. Enfermagem. v.22; n.6, p. 959-965. nov.dez, 2014. Disponível em < http://www.scielo.br/pdf/rlae/v22n6/pt_0104-1169-rlae-22-06-00959.pdf> Acesso em: $13 / 05 / 2017$.

DIAS, M.A.B; DOMINGUES, R.M.S.M; SCHILITHZ, A.O.C; PEREIRA, M.N; DINIZ, C.S.G; BRUM, I.R;MARTINS, A.L; FILHA, M.M.T; GAMA, S.G.N; LEAL, M.C. Incidência do Near Miss materno no parto e pós-parto hospitalar: dados da pesquisa Nascer no Brasil. Cad. Saúde Pública. v.30, p.169-S181, Rio de Janeiro. 2014. Disponível em < http://www.scielo.br/scielo.php?script=sci_arttext\&pid=S0102-311X2014001300022 > Acesso em: 27/05/2017. 
ESSER, M.A.M.S; MAMEDE, F.V; MAMEDE, M.V. The profile of nursing professionals working in maternity wards in Londrina, PR. Rev. Eletr. Enf. v. 14; n.1, p. 133-141. Jan-mar, 2012. Disponível em: https://www.fen.ufg.br/fen_revista/v14/n1/pdf/v14n1a15.pdf Acesso em 23/03/2017.

FEITOSA, M.C; LEITE, I.R.L; SILVA, S.R.F. Demanda de intervenções de enfermagem a pacientes sob cuidados intensivos: NAS - Nursing Activities Score. Esc Anna Nery. v.16, n.4, p.682- 688, out-dez. 2012. Disponível em < http://www.scielo.br/scielo.php?script=sci_arttext\&pid=S1414-81452012000400006 > cesso em: 14/08/2017.

FLETCHER, R.H.; FLETCHER, S.W. Epidemiologia clínica: elementos essenciais. 4. ed., Porto Alegre: Artes Médicas, 2007.

FONSECA, J. J. S. Metodologia da pesquisa científica. Fortaleza: UEC, 2002.

GALVÃO, L.P.L; PEREIRA, F.A; MENDONÇA, C.M.M; MENEZES, F.E.F; GÓIS, K.A.N; RIBEIRO JR; R.F; GURGEL 2 R.Q. The prevalence of severe maternal morbidity and Near Miss and associatedfactors in Sergipe, Northeast Brazil. BMC Pregnancy and Childbirth. 2014. Disponível em < https://bmcpregnancychildbirth.biomedcentral.com/articles/10.1186/1471-2393-14-25> Acesso em: 08/08/2017.

GARDENAL, C.L.C; PARREIRA, I; ALMEIDA, J.M; PEREIRA, V.M. Perfil das enfermeiras que atuam na assistência à gestante, parturiente e puérpera, em instituições de Sorocaba/São Paulo (1999). Rev. Latino-am Enfermagem. Julho-agosto, v.10, n.4, p. 478-84, 2002. Disponível em < http://www.scielo.br/scielo.php?script=sci_arttext\&pid=S0104$11692002000400003 \& \operatorname{lng}=e n \& n r m=i s o \& t \operatorname{lng}=$ pt $>$ Acesso em: 06/07/2017.

GIL, A. C. Como elaborar projetos de pesquisa. - 4. ed. - São Paulo: Atlas, 2007.

INSTITUTO BRASILEIRO GEOGRAFIA E ESTATÍSTICA. Censo 2010. Disponível em <http://www.ibge.com.br > Acesso em 23/02/2017.

LIMA, M.F.G; PEQUENO, A.M.C; RODRIGUES, D.P; CARNEIRO, C; MORAIS, A.P.P; NEGREIROS, F.D.S. Desenvolvendo competências no ensino em enfermagem obstétrica: aproximações entre teoria e prática. Rev Bras Enferm. v.70, n 5, p.1110-6, 2017. Disponível em < http://www.scielo.br/scielo.php?pid=S003471672017000501054\&script=sci_arttext\&tlng=pt > Acesso em: 23/09/2017.

LUZ, A.G; OSIS, M.J.D; RIBEIRO, M; CECATTI, J.G; AMARAL, E. Impact of a nationwide study for surveillance of maternal near-miss on the quality of care provided by participating centers: a quantitative and qualitative approach. BMC Pregnancy and Childbirth. 2014. Disponível em<

https://www.ncbi.nlm.nih.gov/pmc/articles/PMC4234017/ > Acesso em: 09/08/2017.

MASSAROLI, R; MARTINI, J.G; MASSAROLI, A; LAZZARI, D.D; OLIVEIRA, S.N; CANEVER, B.P. Trabalho de enfermagem em unidade de terapia intensiva e sua interface com a sistematização da assistência. Esc. Anna Nery. v.19 n.2, abr-jun, Rio de Janeiro. 2015. Disponível em < http://www.scielo.br/scielo.php?script=sci_arttext\&pid=S1414-81452015000200252 > Acesso em: 25/07/2017.

MORAIS, M.P, MARTINS, J.T; GALDINO, M.J.Q; ROBAZZI, M.L.C.C; TREVISAN, G.S Nurses's work satisfaction in a university hospital. Rev Enferm UFSM. V.6, n.1; p.1-9. Jan/Mar. 2016. Disponível em< https://periodicos.ufsm.br/reufsm/article/view/17766/pdf > Acesso em: 05/09/2017.

NARDELLO, D.M; GUIMARÃES, A.M.D.N; BARRETO, I.D.C; GURGEL, R.Q; RIBEIRO, E.R.O; GOIS, C.F.L. . Óbitos fetais e neonatais de filhos de pacientes classificadas com near miss. Rev Bras Enferm. v. 70, n.1, p.104-11, jan-fev, 2017. Disponível em < http://www.scielo.br/scielo.php?script=sci_arttext\&pid=S003471672017000100104\&lng=en\&nrm=iso\&tlng=pt >. Acesso em: 03/07/2017.

NASCIMENTO, J.M.J; SANTOS, A.A.P; SILVA, F.C.L; SILVA, R.C; LAURINDO, J.A.C; CERQUEIRA, G.S. Os desafios encontrados pelo profissional de enfermagem durante a assistência prestada numa maternidade pública. EFDeportes.com Revista Digital. Buenos Aires, v19, n.195, agosto de 2014. Disponível em: < http://www.efdeportes.com/efd195/profissional-de-enfermagem-numa-maternidade-publica.htm >. Acesso em: 25/08/2017 
OLIVEIRA, E.M; SPIRI, W.C. Dimensão pessoal do processo de trabalho para enfermeiras de Unidades de Terapia Intensiva. Acta Paul Enferm. v.24, n.4, p.550-555, 2011. Disponível em <

http://www.scielo.br/scielo.php?script=sci_arttext\&pid=S0103-21002011000400016> Acesso em: 03/07/2017.

OLIVEIRA, L. C; COSTA , A. A. Maternal near miss in the intensive care unit: clinical and epidemiological aspects. Rev Bras Ter Intensiva, v. 27, n. 3, p. 220-7,2015. Disponível em<

https://www.ncbi.nlm.nih.gov/pmc/articles/PMC4592115/> Acesso em: 23/04/2017.

OLIVEIRA, L.C; COSTA, A.A.R. Fetal and neonatal deaths among cases of maternal near miss. Rev Assoc Med Bras. v. 59, n.5, p. 487 - 494. 2013. Disponível em: <https://www.sciencedirect.com/science/article/pii/s0104423013001528?via\%3Dihub> Acesso em: 23/04/2017.

OUD , L. Epidemiology of Pregnancy Associated ICU Utilization in Texas: 2001 - 2010. J Clin Med Res, v. 9, n.2, p. 143-53, 2017. Disponível em < https://www.ncbi.nlm.nih.gov/pmc/articles/PMC5215018/> Acesso em: 03/10/2017.

ROSENDO, T.M.S.S; RONCALLI, A.G. Prevalência e fatores associados ao Near Miss Materno: inquérito populacional em uma capital do Nordeste Brasileiro. Ciência \& Saúde Coletiva. v.20, n.4, p.1295-1304. 2015. Disponível em < http://www.scielo.br/scielo.php?script=sci_arttext\&pid=S141381232015000401295\&lng=en\&nrm=iso\&tlng=pt> Acesso em: 27/03/2017.

RUDEY, E.L; CORTEZ, L.E.R; YAMAGUCHI, M.U. Identificação de near miss materno em unidade de terapia intensiva. Revista Saúde e Pesquisa. v. 10, n. 1, p. 145-155, jan./abr. 2017. Disponível em < http://periodicos.unicesumar.edu.br/index.php/saudpesq/article/view/5813/3015> Acesso em: 14/06/2017.

SERGIPE. Maternidade Nossa Senhora de Lourdes registrou mais de 16 mil atendimentos no ano passado. Disponível em: < http://www.agencia.se.gov.br/noticias/saude/maternidade-nossa-senhora-delourdes-registrou-mais-de-16-mil-atendimentos-no-ano-passado >. Acesso em: 02/03/2017.

SILVEIRA, R.S; FUNCK, C.R; LUNARDI, V.L; AVILA, L.I; LUNARDI FILHO, W.D; VIDAL, D.A.S. Percepção dos trabalhadores de enfermagem acerca da satisfação no contexto do trabalho na UTI. Enferm Foco. v.3; n.2, p.93-96, 2012. Disponível em http://revista.cofen.gov.br/index.php/enfermagem/article/view/262. Acesso em: 23/03/2017.

TUNÇALP, O; HINDIN, M.J; SOUZA, J.P; CHOU, D; SAY, L. The prevalence of maternal Near Miss: a systematic review. BJOG. v.119, p. 653-661. 2012. Disponível em:<

https://obgyn.onlinelibrary.wiley.com/doi/pdf/10.1111/j.1471-0528.2012.03294.x >. Acesso em: 23/02/2017

VILLELA, L.C.M; GALASTRO, E.P; FREITAS, M.E.A; SANTOS, M.S.G; NOTARO, K.A.M. Tempo de atuação do profissional enfermeiro - Minas Gerais. Enfermagem em Foco. v.2; n.4, p248-250. 2011. Disponível em < http://revista.cofen.gov.br/index.php/enfermagem/article/view/194> Acesso em: 30/05/2017.

WORLD HEALTH ORGANIZATION. Trends in maternal mortality: 1990-2008. 2010. Disponível em: < https://www.unfpa.org/sites/default/files/pub-pdf/trends_matmortality90-08.pdf> Acesso em: 28/02/2017.

WORLD HEALTH ORGANIZATION. World Health Statistics 2009. Disponível em < http://www.who.int/whosis/whostat/EN_WHS09_Full.pdf?ua=1 > . Acesso em: 04/05/2017.

\section{(c)) EY}

Este trabalho está licenciado com uma Licença Creative Commons - Atribuição 4.0 Internacional. 